\title{
Preliminary estimation
} of high-frequency $(4-20 \mathrm{~Hz})$ energy released from the 2016 Kumamoto, Japan, earthquake sequence

\author{
Kaoru Sawazaki ${ }^{*}$, Hisashi Nakahara ${ }^{2}$ and Katsuhiko Shiomi ${ }^{1}$
}

\begin{abstract}
We estimate high-frequency $(4-20 \mathrm{~Hz}$ ) energy release due to the 2016 Kumamoto, Japan, earthquake sequence, within a time period from April 14 to 26 through envelope inversion analysis applied to the Hi-net continuous seismograms. We especially focus on energy releases after each of the April $14 M_{\mathrm{JMA}} 6.5$ and the April $16 M_{\mathrm{JMA}} 7.3$ earthquakes. The cumulative energy release from aftershocks of the April 14 event reaches $60 \%$ of that from the April 14 event itself by the lapse time of $27 \mathrm{~h}$ (pre-April 16 period). On the other hand, the cumulative energy release from aftershocks of the April 16 event reaches only 11 and 13\% of that from the April 16 event itself by the lapse times of $27 \mathrm{~h}$ and 10 days (post-April 16 period), respectively. This discrepancy in the normalized cumulative energy release (NCER) indicates that the April 14 event was followed by much larger relative aftershock productivity than the April 16 event. Thus, NCER would provide information that reflects relative aftershock productivity and ongoing seismicity pattern after a large earthquake. We also find that the temporal decay of the energy release rate obeys the power law. The exponent $p_{E}$ of the power-law decay is estimated to be 1.7-2.1, which is much larger than the typical $p$ value of the Omori-Utsu law: slightly larger than 1 . We propose a simple relationship given by $p_{\mathrm{E}}=\beta p / b$, where $p$ value, $b$ value of the Gutenberg-Richter law, and $\beta$ value of the magnitude-energy release relationship are combined.
\end{abstract}

Keywords: 2016 Kumamoto earthquake sequence, Aftershocks, High-frequency energy release, Normalized cumulative energy release

\section{Introduction}

At 21:26 (JST) on April 14, 2016, an $M_{\text {JMA }} 6.5$ earthquake (hereafter, April 14 event) occurred in Kumamoto district, center of Kyushu Island, Japan, and was followed by a significant number of earthquakes including the $M_{\mathrm{JMA}} 6.4$ event at 00:03 on April 15. Twenty-eight hours after the April 14 event, an $M_{\mathrm{IMA}} 7.3$ earthquake (hereafter, April 16 event) occurred in the same district at 01:25 on April 16. This event triggered widespread seismicity not only in Kumamoto district but also in northeastern distant areas like Aso district and Oita prefecture,

\footnotetext{
*Correspondence: sawa@bosai.go.jp

${ }^{1}$ National Research Institute for Earth Science and Disaster Resilience, Tsukuba, Japan

Full list of author information is available at the end of the article
}

and was also followed by large amount of earthquakes. Through this earthquake sequence, in total 50 people were directly killed due to collapse of buildings, mudflows or landslides, and so on (Cabinet Office, Government of Japan 2016).

One of the most important information for the public after a large earthquake is fast and accurate aftershock forecasting; particularly, people worry about whether comparable or even larger earthquakes would follow in the near future or not. For the Kumamoto earthquake sequence, the April $14 M_{\mathrm{JMA}} 6.5$ event had been considered to be the "mainshock" until the larger one $\left(M_{\mathrm{JMA}} 7.3\right)$ occurred on April 16: The original expectation was eventually wrong. In Japan, specific aftershock forecasting is usually announced at least 1 day after the occurrence of a large earthquake because it takes usually more than 1 day 
before catalog of the aftershocks becomes available for the forecast. Detection of earthquakes occurring within early lapse times (within a few hours in general) after a large event is generally very difficult because waveforms of many earthquakes occurring within a short time interval tend to overlap in seismograms, and conventional detection techniques using $\mathrm{P}$ - and/or S-wave picking become unavailable. Lack of the early earthquake catalog is one reason why the aftershock forecasting takes long time before it is announced.

To overcome this defect, some studies use an incomplete early aftershock catalog to estimate the parameter that represents the catalog's incompleteness at the same time with other parameters that control the Omori-Utsu (Utsu 1961) and the Gutenberg-Richter (Gutenberg and Richter 1944) laws (e.g., Omi et al. 2015). More recently, Omi et al. (2016) pointed out that even automatically determined earthquake catalog (thus incompleteness and uncertainty are much serious than final catalog) is available for the aftershock forecasting with a performance comparable to the case of using the final catalog. Another strategy that can improve the early aftershock forecasting is to utilize continuous seismograms directly (e.g., Sawazaki and Enescu 2014; Lippiello et al. 2016). Since this technique can detect energy release from all earthquakes occurring in each consequent time interval, the misdetection should not be a problem in theory.

In this study, we apply the method by Sawazaki and Enescu (2014) with moderate correction to the Hi-net (operated by National Research Institute for Earth Science and Disaster Resilience, NIED) continuous seismograms and estimate high-frequency $(4-20 \mathrm{~Hz})$ energy release from the Kumamoto earthquake sequence. Particularly, we focus on how the energy release in pre-April 16 period (21:26, April 14-01:25, April 16) differs from that in post-April 16 period (01:25, April 16-01:25, April 26) and discuss the possibility of forecasting the April 16 event before it occurred.

\section{Data and method Data processing}

We use 15 Hi-net continuous seismograms recorded in Kyushu Island, Japan, as shown in Fig. 1, where the analyzed time period begins at 21:26, April 14 (origin time of the April 14 event), and ends at 01:25, April 26 (10 days after the April 16 event). Each Hi-net station is composed of three-component high-sensitivity velocity seismometers installed at the depth greater than $100 \mathrm{~m}$, which is originally designed to detect small earthquakes with high signal-to-noise ratio. The sampling frequency of the $\mathrm{Hi}$-net record is $100 \mathrm{~Hz}$, and the recording system response is flat from 1 to about $30 \mathrm{~Hz}$ at least. See Okada et al. (2004) and Obara et al. (2005) for detail of the Hinet recording system.

After removing average and linear trend of the $\mathrm{Hi}$ net seismogram, we apply the 4- to $20-\mathrm{Hz}$ Butterworth band-pass filter to the original velocity seismogram and compute squared sum of the three components. The $4-20 \mathrm{~Hz}$ is selected because the signal-to-noise ratio of the seismograms is high at this frequency range. Then we multiply mass density $2800 \mathrm{~kg} / \mathrm{m}^{3}$ to the record and compute average energy density every $1 \mathrm{~s}$. The obtained seismogram envelope trace at each station is normalized by each local site amplification factor estimated by the codanormalization method (Philips and Aki 1986), where the station N.KHKH is selected as the reference station because this site is characterized by relatively high $V_{\mathrm{P}}$ and $V_{\mathrm{S}}\left(V_{\mathrm{P}}=4.2 \mathrm{~km} / \mathrm{s}\right.$ and $V_{\mathrm{S}}=1.9 \mathrm{~km} / \mathrm{s}$ at $100 \mathrm{~m}$ depth $)$ according to the well-logging data provided by NIED. Then, we divide the envelope by the global site amplification factor (Sawazaki and Enescu 2014) of 3.7 considering reflection of incident wave on the ground surface and difference of $V_{\mathrm{S}}$ at the source $(3.5 \mathrm{~km} / \mathrm{s})$ and that at the reference station $(1.9 \mathrm{~km} / \mathrm{s})$. See Sawazaki and Enescu (2014) for detail of correction of the site amplification factor.

At some Hi-net stations, amplitude of the original velocity seismogram is saturated due to strong ground motion. Shiomi et al. (2005) pointed out that the Hi-net record is overlapped by high-frequency pulse noise when the stroke amplitude of the pendulum exceeds a threshold value $(0.09$ and $0.15 \mathrm{~cm}$ for horizontal and vertical components, respectively) and moving direction of the pendulum is changed suddenly. By comparing the $\mathrm{Hi}$-net records and the colocated KiK-net strong motion records, we found that the contamination of the high-frequency noise is not negligible for six earthquakes with the magnitude larger than 5.8. For these events, we replace the saturated $\mathrm{Hi}$-net records by the KiK-net records obtained at the same location. For the replacement, we first integrate the KiK-net accelerogram to velocity after applying the $0.1-\mathrm{Hz}$ high-pass filter. Then we apply the same data processing as that applied to the Hi-net records and obtain the non-saturated envelope trace.

Figure 2 shows the observed envelope traces (black) obtained in the first $1000 \mathrm{~s}$ after the April 14 event. Many peaks follow after the largest peak associated with the 21:26 $M_{\mathrm{JMA}} 6.5$ event. The average amplitude does not decay to the ambient noise level through the 1000-s duration. This indicates that waveforms from many earthquakes are overlapped in the seismograms within early lapse times after the April 14 event. 


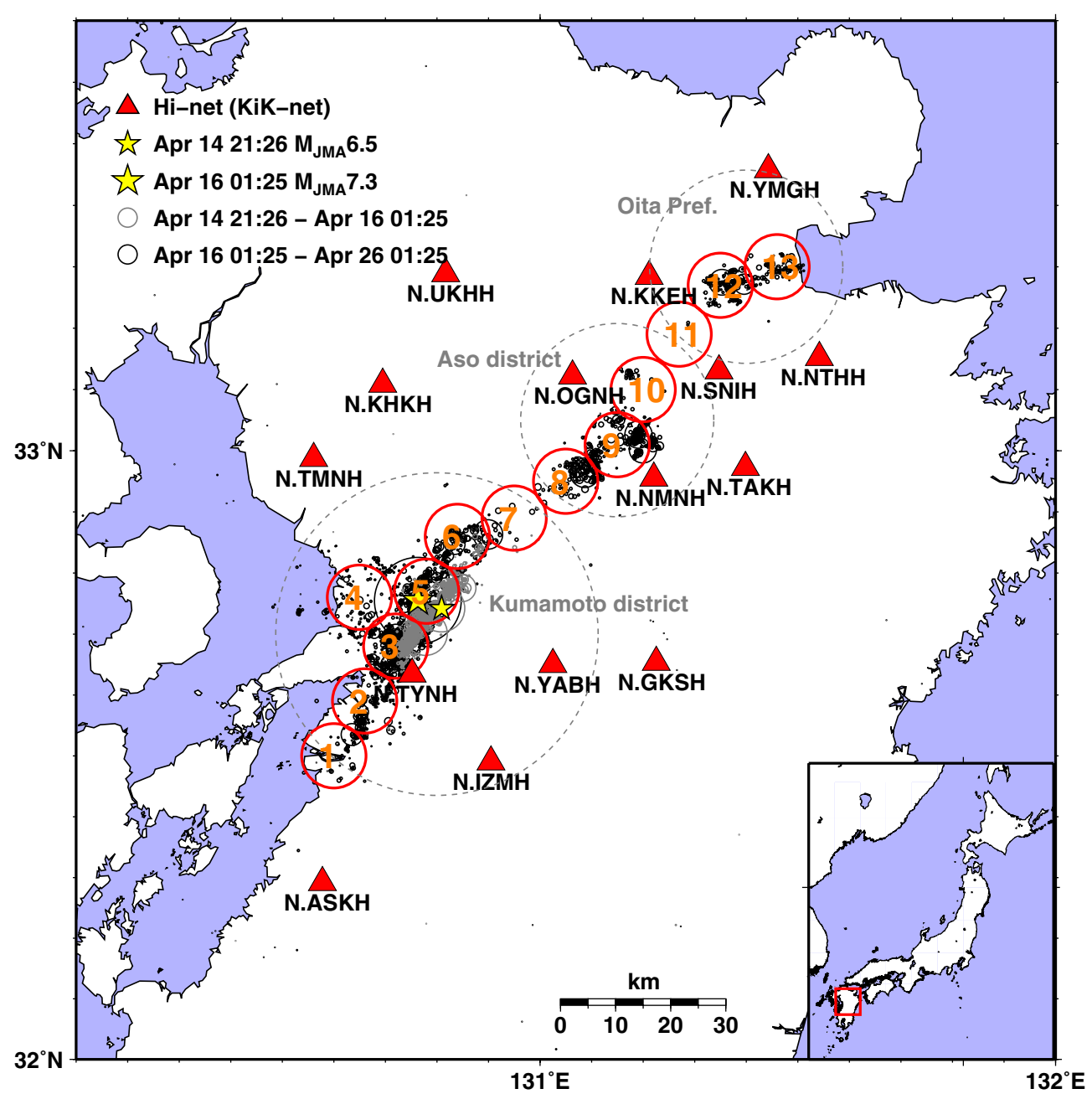

Fig. 1 Map of the earthquake distribution associated with the 2016 Kumamoto earthquake sequence. The black and gray circles represent the earthquakes occurring within pre- and post-April 16 periods, respectively. The stars represent the epicenters of the April 14 (small star) and the April 16 (large star) events. The triangles and the red circles represent the Hi-net (KiK-net) stations and the source-grids for the energy release (located at $10 \mathrm{~km}$ depth) used for the envelope inversion analysis, respectively. The red rectangle in the inserted map represents the studied area

\section{Envelope inversion}

We apply the envelope inversion technique developed by Sawazaki and Enescu (2014) to the observed envelope traces. The theoretical seismogram envelope $E_{i}$ at the $i$-th station is described by

$$
E_{i}(t)=S_{i} \int_{0}^{t} G_{i, j}(t-\tau) W_{j}(\tau) \mathrm{d} \tau,
$$

where $S_{i}, G_{i, j}$, and $W_{j}$ represent the site amplification factor at the $i$-th station, envelope Green's function, and energy release at the $j$-th source-grid, respectively. By using properly selected site amplification factors and the envelope Green's functions, we invert for energy release $W$ in the target frequency range of $4-20 \mathrm{~Hz}$. To estimate
$W$ at each time-grid, we first select location of the energy release from the employed 13 source-grids (red circles in Fig. 1), where depth of each grid is set to be $10 \mathrm{~km}$ considering the distribution of the aftershocks. Here, only one source-grid is selected as the energy release location for each time-grid. Then, by fixing the selected energy release location, we estimate the amount of energy release that matches the amplitudes of the observed envelopes. This procedure is carried out for all time-grids successively. See Sawazaki and Enecsu (2014) for detail of the envelope inversion scheme.

The original scheme of Sawazaki and Enescu (2014) is moderately corrected as follows. First, we use the hybrid synthetic envelope as the envelope Green's function, where direct and coda parts of the envelope are 


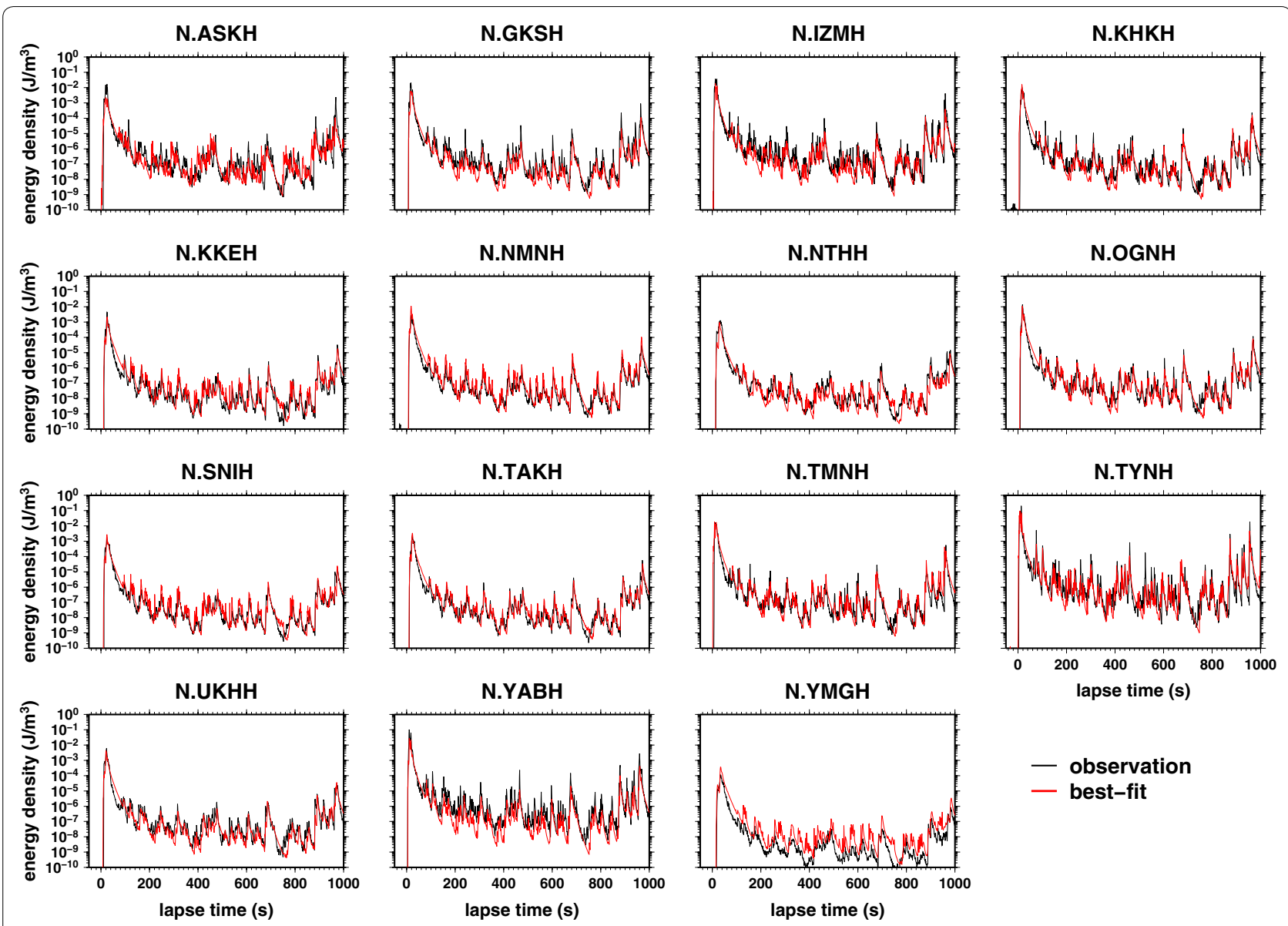

Fig. 2 Black and red curves represent the observed and the best-fit energy density envelopes (4-20 Hz) for the first $1000 \mathrm{~s}$ after the April 14 event

synthesized on the basis of the forward scattering approximation (Shishov 1974) and the multiple isotropic scattering model (Paasschens 1997), respectively. This hybrid envelope better describes the whole envelope shape than the isotropic scattering model-based envelope used in Sawazaki and Enescu (2014) does. The synthesis of the hybrid envelope is summarized in Appendix 1. We synthesize not only S-wave but also P-wave envelope because amplitude of $\mathrm{P}$-wave is not negligible in logarithmic scale. Second, we determine the energy release location using the theoretical S-wave peak arrival times. This strategy is different from that adopted by Sawazaki and Enescu (2014) who used theoretical peak amplitudes for estimation of the energy release location. The difference between the previous and the newly developed schemes for the location determination is explained in Appendix 2. Through these corrections, accuracy of the energy release location is considerably improved (Sawazaki 2016).

We determine the parameters that characterize the subsurface structure as follows: $V_{\mathrm{P}}=6.1 \mathrm{~km} / \mathrm{s}$
$V_{\mathrm{S}}=3.5 \mathrm{~km} / \mathrm{s}, \varepsilon$ (RMS fractional velocity fluctuation for a 3-D Gaussian-type random inhomogeneous media $)=0.12$, $a$ (correlation length) $=5 \mathrm{~km}, g_{0}$ (scattering coefficient) $=1.0 \times 10^{-2} \mathrm{~km}^{-1}$, and $Q_{i}^{-1}$ (intrinsic absorption factor) $=1.2 \times 10^{-3}$. These values are determined from inspection of small earthquake records and previous studies by Carcole and Sato (2010), Sato et al. (2012), and so on. We use the theoretical envelopes synthesized using these parameters as the envelope Green's function.

\section{Result of the envelope inversion analysis}

Figure 2 shows the comparison between the observed (black) and the best-fit (red) envelopes for the first $1000 \mathrm{~s}$ after the April 14 event. The fitness is generally good except for a few overestimated (e.g., N.YMGH) and underestimated (e.g., N.YABH) stations. These misfits are probably due to local difference in the scattering and intrinsic absorption factors and/or directionality due to radiation pattern, which are not reflected in the used envelope Green's function. 
Figure 3a shows the estimated 4- to $20-\mathrm{Hz}$ energy release rate from 21:26, April 14, to 01:25, April 26. Overall feature of the energy release rate is characterized by two large peaks that correspond to the April $14 M_{\mathrm{IMA}} 6.5$ and the April $16 M_{\mathrm{JMA}} 7.3$ events, and the gradual decay after these two major events. The red line $\left(4.1 \times 10^{3} \mathrm{~J} / \mathrm{s}\right)$ indicates five times as large energy release rate as that in the time period when background ambient noise amplitude dominants, above which the energy is considered to be mostly excited by earthquakes. The energy from the Kumamoto earthquake sequence is much beyond the energy from the background noise even at the lapse time of 10 days after the April 16 event.

Figure $3 \mathrm{~b}$ demonstrates the zoomed energy release rate around the origin time (0 s) of the April 14 (black) and the April 16 events (red). Integrating the energy release rate from -5 to $25 \mathrm{~s}$, we estimate the cumulative energy release from the April 14 and the April 16 events as $2.7 \times 10^{12} \mathrm{~J}$ and $2.1 \times 10^{13} \mathrm{~J}$, respectively, in $4-20 \mathrm{~Hz}$ : The latter released eight times larger energy than the former.

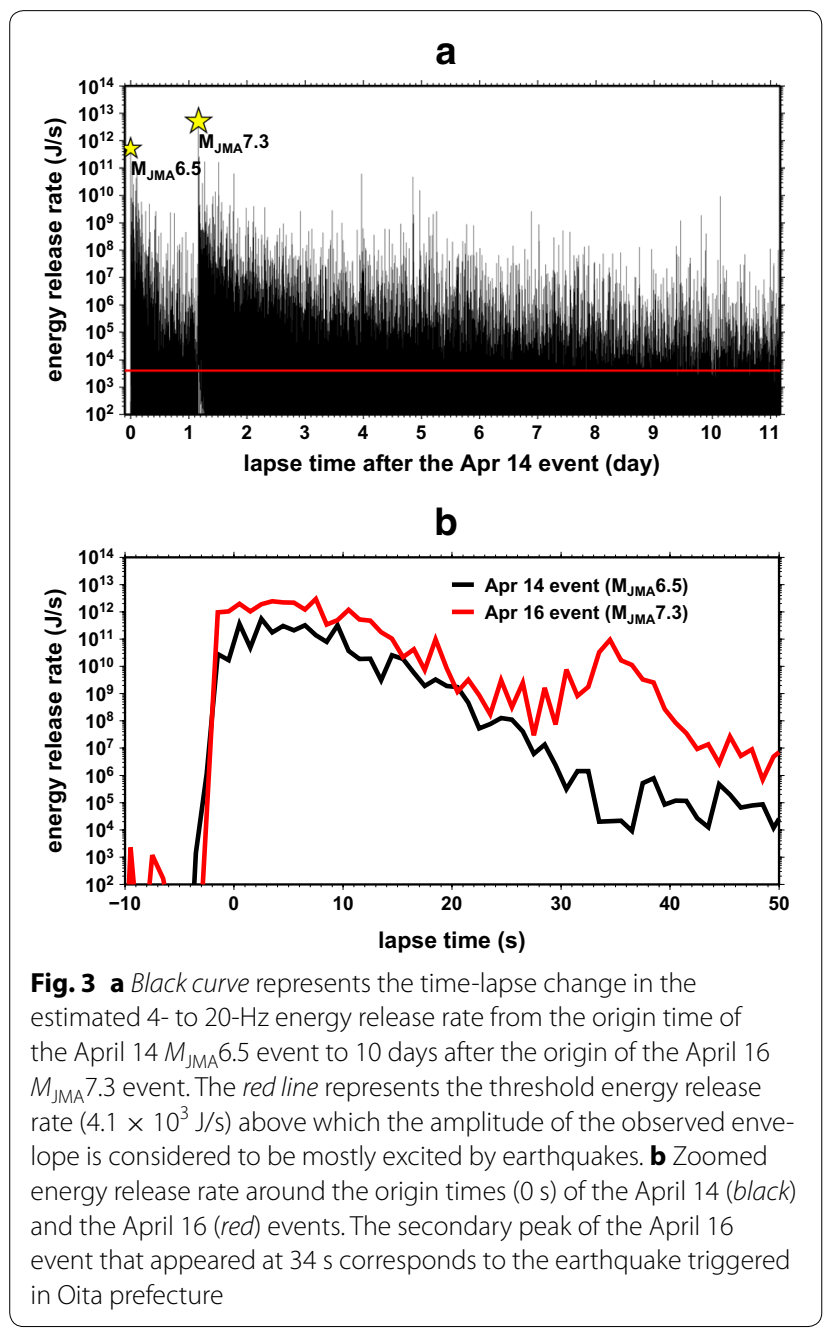

It is well known that the number of aftershocks usually decays following power law after the mainshock (Utsu 1961). Considering this point, we approximate the energy release rate $\dot{W}$ by a power-law function given by

$$
\dot{W}(t)=\frac{\dot{W}_{0}}{\left(1+t / c_{\mathrm{E}}\right)^{p_{\mathrm{E}}}},
$$

where $\dot{W}_{0}, c_{\mathrm{E}}$, and $p_{\mathrm{E}}$ are the initial energy release rate at lapse time $t=0$, the onset time of the power-law decay (analogous to $c$ value of the Omori-Utsu law), and the exponent of the power-law decay (analogous to $p$ value of the Omori-Utsu law), respectively. We fix $c_{\mathrm{E}}=50 \mathrm{~s}$ considering previous studies (e.g., Enescu et al. 2009) and estimate $\dot{W}_{0}$ and $p_{\mathrm{E}}$ by applying the least-squares method to the obtained energy release rate in logarithmic scale.

Figure 4 shows 1 -h average of the estimated energy release rate (thin lines) and the best-fit power-law decay curves for the April 14 (black) and the April 16 (red) events, where zero-lag time corresponds to each origin time. Here, we use the energy releases from the sourcegrids numbered by 1-8 (Fig. 1) because the energy releases from other northeastern grids should be caused by the remotely triggered seismicity after the April 16 event, which could be characterized by different timelapse decay curve. Although the fluctuation is strong, the average feature of the energy release rate is characterized by the power-law decay for both pre- and post-April 16 periods. The estimated values and ranges $( \pm 1 \sigma)$ are

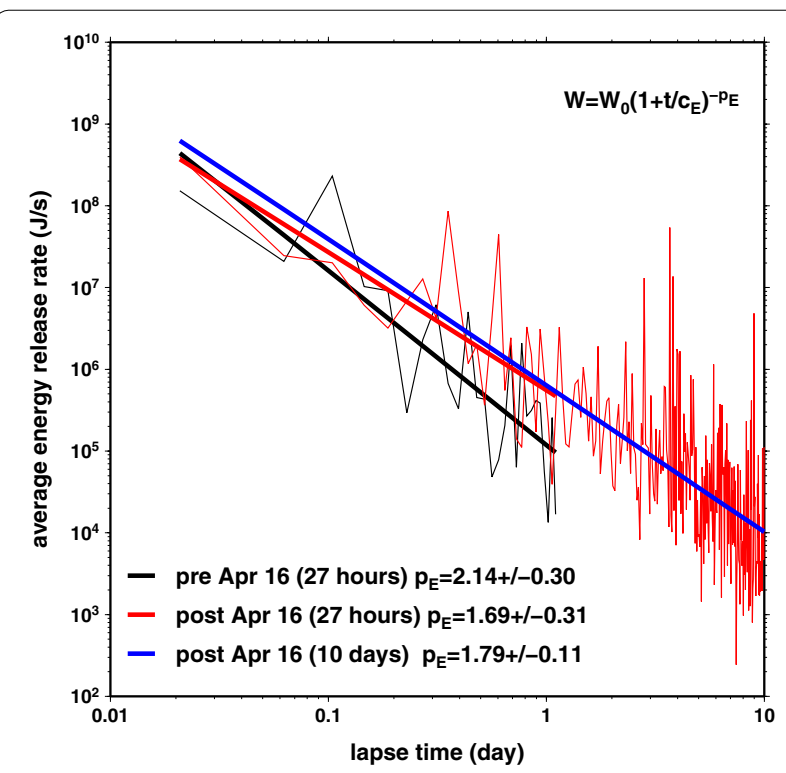

Fig. 4 Thin curves represent 1-h average of the 4- to 20-Hz energy release rate for pre (black)- and post (red)-April 16 periods. The thick lines are regression lines computed by fitting Eq. (2) to the average energy release rates. Two regression lines are fitted for the post-April 16 period using different time durations (red $27 \mathrm{~h}$, blue 10 days) 
$\dot{W}_{0}=(0.14-7.1) \times 10^{12}(\mathrm{~J} / \mathrm{s})$ and $p_{\mathrm{E}}=2.14 \pm 0.30$ for the pre-April 16 period (black thick line) and $\dot{W}_{0}=(0.022-$ 1.3) $\times 10^{12}(\mathrm{~J} / \mathrm{s})$ and $p_{\mathrm{E}}=1.69 \pm 0.31$ for the post-April 16 period (red thick line). If we extend the post-April 16 period to 10 days, we obtain $\dot{W}_{0}=(0.16-1.0) \times 10^{12}(\mathrm{~J} / \mathrm{s})$ and $p_{\mathrm{E}}=1.79 \pm 0.11$ (blue line). It is interesting that the estimated $p_{\mathrm{E}}$ values are significantly larger than typical $p$ value of the Omori-Utsu law, which is slightly larger than 1 in general. Interpretation of the obtained power-exponent $p_{\mathrm{E}}$ is discussed in chapter 4 .

Figure 5 shows the correlogram between the estimated energy release and $M_{\mathrm{JMA}}$. The energy release $W_{\mathrm{obs}}$ from each earthquake is computed by integrating the energy release rate within $\pm 2 \mathrm{~s}$ of each origin time. We use the empirical relationship given by

$$
\log W_{\mathrm{obs}}=\alpha+\beta M_{\mathrm{JMA}}
$$

to approximate the energy-magnitude relationship, where $\alpha$ and $\beta$ are constant values (e.g., Utsu 2001). Using the least-squares method, we estimate $\alpha=2.8$ and $\beta=1.6$ in the range of $1.5 \leq M_{\mathrm{IMA}}<4.5$ (red line in Fig. 5). This regression line underestimates the magnitude when $M_{\text {IMA }}$ is larger than about 4.5. This underestimation would be mainly due to the difference in the used frequency ranges: We use the frequency range of $4-20 \mathrm{~Hz}$ to obtain $W_{\mathrm{obs}}$, while $M_{\mathrm{IMA}}$ is determined using frequencies down to $1 \mathrm{~Hz}$ or lower. The corner frequency of an M4.5 earthquake is usually lower than $4 \mathrm{~Hz}$ (e.g., Eshelby 1957; Madariaga

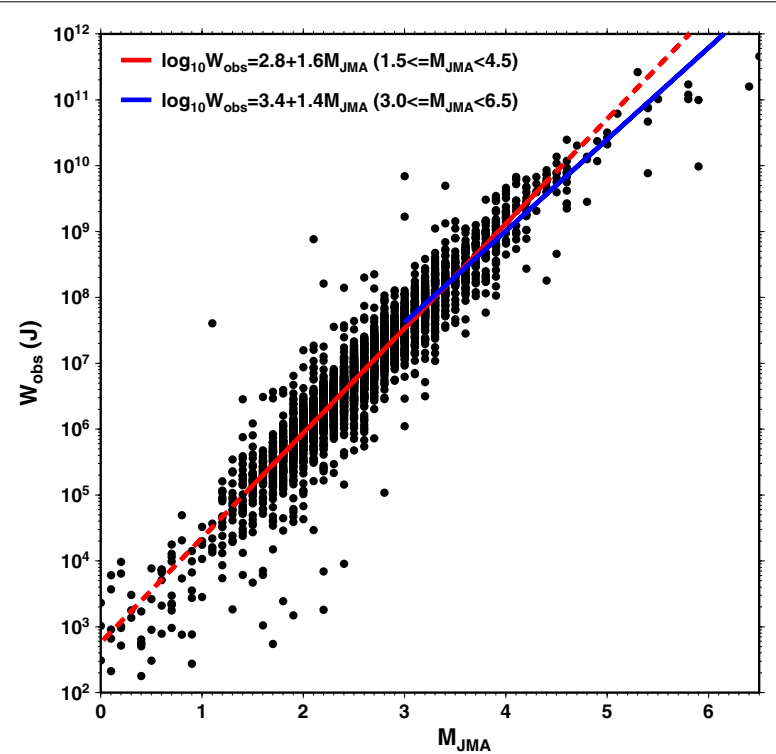

Fig. 5 Black circles represent the correlogram between the 4- and 20-Hz energy release $\left(W_{\mathrm{obs}}\right)$ and $M_{\mathrm{JMA}}$. The regression lines are obtained by fitting Eq. (3) to the correlogram, where the magnitude ranges used for the fitting are $1.5 \leq M_{\mathrm{JMA}}<4.5$ and $3.0 \leq M_{\mathrm{JMA}}<6.5$ for the red and blue lines, respectively
1976). Therefore, $4-$ to $20-\mathrm{Hz}$ range cannot sense the large energy release around the corner frequency sufficiently for earthquakes larger than M4.5, while $M_{\mathrm{IMA}}$ can better reflect the energy release from these larger earthquakes. If we fit the regression line to the range of $3.0 \leq M_{\mathrm{IMA}}<6.5$, $\alpha=3.4$ and $\beta=1.4$ are estimated (blue line in Fig. 5).

Figure $3 \mathrm{~b}$ shows a secondary peak at the lapse time of $34 \mathrm{~s}$ after the April 16 event, which corresponds to the energy release from the earthquake triggered at Oita prefecture (close to the number 12 source-grid in Fig. 1). Magnitude of this earthquake is estimated to be 5.6 from Eq. (3) using $\alpha=3.4$ and $\beta=1.4$. This magnitude is similar to $M_{\mathrm{JMA}} 5.7$, which had not been reported until a careful survey completes: Detection of this earthquake took a long time because seismograms are partially overlapped by coda wave of the April 16 event. Our envelope inversion technique can determine magnitude of this earthquake correctly in quasi real-time without disturbance by the coda wave.

Figure 6 shows the spatial distribution of the 4- to $20-\mathrm{Hz}$ energy release within different time periods. The panels entitled by "Apr 14 21:26 $M_{\mathrm{JMA}} 6.5$ " and "Apr 16 01:25 $M_{\mathrm{IMA}} 7.3$ " demonstrate the distribution of energy release from each earthquake, of which the energy release is obtained by integrating the energy release rate from -5 to $25 \mathrm{~s}$ after each earthquake origin time. Other panels demonstrate the distribution of cumulative energy release within the entitled time period. The large energy release is concentrated to the central to southwest source-grids with numbers 4-8 (see grid numbers in Fig. 1) for the April 14 and the April 16 events. We note that our inversion scheme selects only single energy release location at each 1-s time-grid, which can cause a biased distribution in energy release if energies are released at the same time at different source-grids. This bias may not be negligible during a large earthquake whose rupture propagates across multiple source-grids, which may be the reason why no energy is released at source-grids 1-3 during the April 14 and the April 16 earthquakes. A remarkable amount of energy release appears in the northeastern source-grids after the April 16 event, which corresponds to the remotely triggered seismicity at Aso district and Oita prefecture. The energy release is large at almost all source-grids within 1 day after the April 16 event. After that, the energy release gradually decays, but is occasionally activated regionally corresponding to occurrence of large earthquakes (see JMA unified hypocenters drawn by blue circles).

\section{Discussion}

\section{Consistency between the estimated energy release rate and the aftershock catalog}

The estimated $p_{\mathrm{E}}$ values (see Fig. 4) are significantly larger than the well-reported $p$ value of the OmoriUtsu law: around 1 or slightly larger in many cases. This 


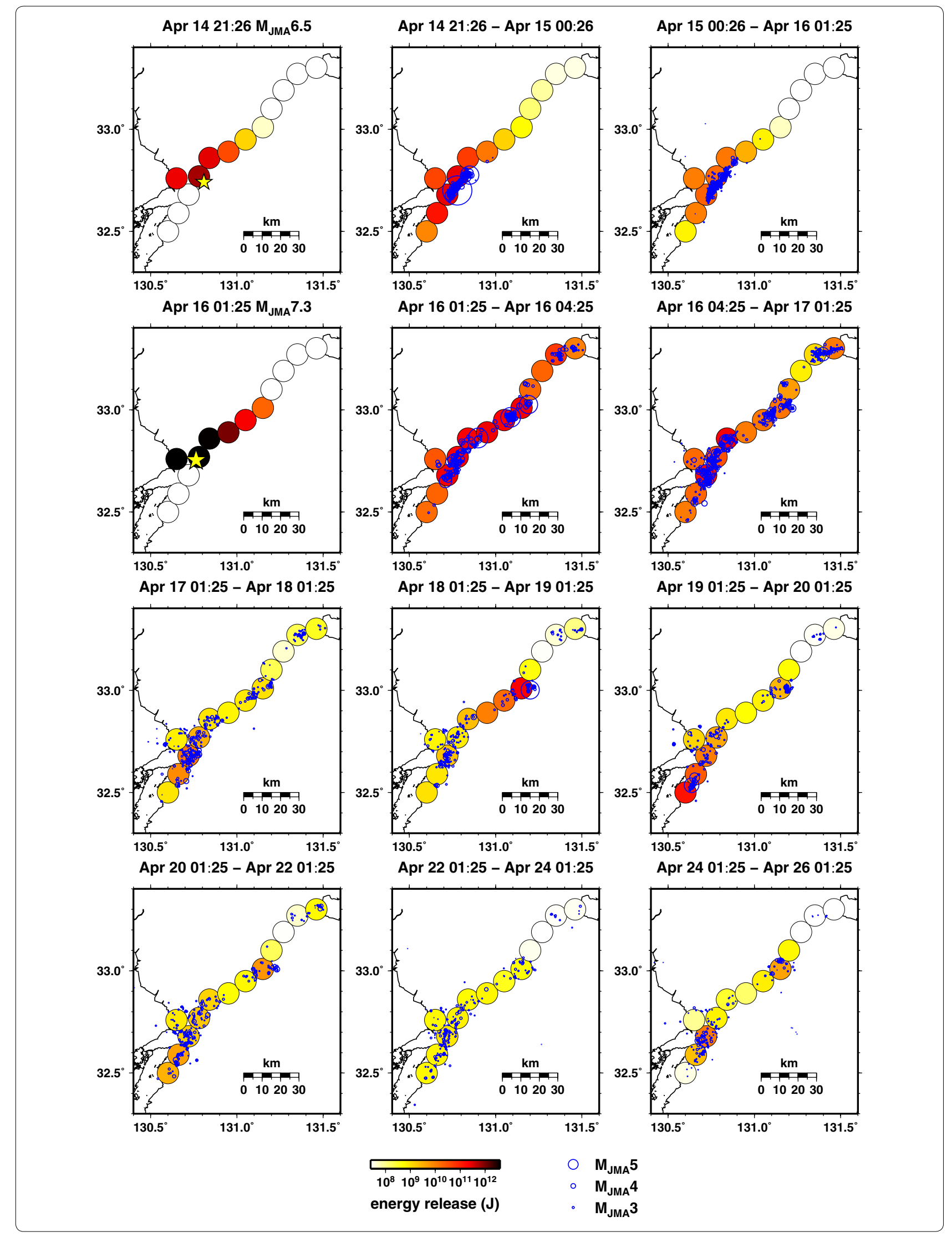


(See figure on previous page.)

Fig. 6 Colored circles represent the distribution of the 4- to 20-Hz energy release in each time period. The panels entitled by "April 14 21:26 $\mathrm{M}_{\mathrm{JMA}} 6.5$ " and "April $1601: 25$ M JMA.7.3" represent the energy release within - 5 to $25 \mathrm{~s}$ after the origin times of the April 14 and April 16 events, respectively. The blue circles represent the JMA unified hypocenters detected in each time period

discrepancy between the estimated $p_{\mathrm{E}}$ and $p$ values could be explained as follows. Combination of the Omori-Utsu law and the Gutenberg-Richter law provides

$$
N(M, t) \mathrm{d} M \mathrm{~d} t=\frac{K}{(t+c)^{\mathrm{p}}} 10^{-\mathrm{bM}} \mathrm{d} M \mathrm{~d} t,
$$

where $N(M, t) \mathrm{d} M \mathrm{~d} t$ is expected number of earthquakes with the magnitude between $M$ and $M+\mathrm{d} M$ at the lapse time between $t$ and $t+\mathrm{d} t$. Using the energy releasemagnitude relationship given by Eq. (3) and the expected number of earthquakes given by Eq. (4), we obtain the energy release $\dot{W}(t) \mathrm{d} t$ from all earthquakes occurring within $t$ and $t+\mathrm{d} t$ as

$$
\dot{W}(t) \mathrm{d} t=\int_{-\infty}^{M_{\max }(t)} \frac{K}{(t+c)^{p}} 10^{-\mathrm{bM}} 10^{\alpha+\beta \mathrm{M}} \mathrm{d} M \mathrm{~d} t,
$$

where $M_{\max }(t)$ means the expected magnitude of the largest earthquake that occurs within $t$ and $t+\mathrm{d} t$. If $\beta-b>0$ (satisfied for most cases), Eq. (5) is integrable and gives

$$
\dot{W}(t) \mathrm{d} t=\frac{K 10^{\alpha}}{(t+c)^{p}} \frac{1}{(\beta-b) \ln 10} \exp \left[(\beta-b) \ln 10 \cdot M_{\max }(t)\right] \mathrm{d} t .
$$

In this study, $M_{\max }(t)$ is determined so that it satisfies the expected number of earthquakes with the magnitude between $M_{\max }(t)$ and $M_{\max }(t)+\mathrm{d} M$ in the lapse time between $t$ and $t+\mathrm{d} t$ becomes $\mathrm{d} M \mathrm{~d} t\left(N\left(M_{\max }(t), t\right)=1\right)$. Therefore, Eq. (4) gives

$$
\begin{array}{r}
\mathrm{d} M \mathrm{~d} t=\frac{K}{(t+c)^{p}} 10^{-b M_{\max }(t)} \mathrm{d} M \mathrm{~d} t \\
M_{\max }(t)=-\frac{1}{b} \log \frac{(t+c)^{p}}{K}
\end{array}
$$

Although Eq. (7) is given somewhat arbitrarily, we confirmed that this formula actually describes the observed time-lapse decay of $M_{\max }$ well for the Kumamoto earthquake sequence. By substituting the derived $M_{\max }(t)$ in Eq. (6), we obtain

$$
\dot{W}(t) \mathrm{d} t=\frac{10^{\alpha} K^{\frac{\beta}{b}}}{(\beta-b) \ln 10 \cdot(t+c)^{\frac{\beta}{b} p}} \mathrm{~d} t .
$$

By comparing Eqs. (2) and (8), the parameter values are given by

$$
p_{\mathrm{E}}=\frac{\beta}{b} p, \quad c_{\mathrm{E}}=c, \quad \dot{W}_{0}=\frac{10^{\alpha} K^{\frac{\beta}{b}}}{(\beta-b) c^{\beta p / b} \ln 10} .
$$

Equation (8) indicates that the energy release rate decays following the power law with the power exponent given by $p_{\mathrm{E}}=\beta \mathrm{p} / b$, not by $p$.

To examine consistency of the estimated $p_{\mathrm{E}}$ value with the $b$ and $p$ values, we analyze the JMA unified hypocenter catalog (as of September 15, 2016). Figure 7 shows the magnitude-frequency diagram obtained for three different time periods. Considering the magnitude of detection threshold, we use the earthquakes that satisfy $M_{\mathrm{JMA}} \geq 3.0$ for estimation of the $b$ value. Applying the least-squares method to the magnitude-frequency diagram in the logarithmic scale, we estimate $b=0.71 \pm 0.04, b=0.74 \pm 0.06$, and $b=0.80 \pm 0.04$ in the pre-April 16 period (black line), the post-April 16 period for $27 \mathrm{~h}$ long (red line), and the post-April 16 period for 10 days long (blue line), respectively. We use the least-squares method rather than the conventional maximum-likelihood method because $b, p$, and $p_{\mathrm{E}}$ values should be estimated using the same method for a comparison. The $b$ value obtained in the pre-April 16 period is slightly smaller than that obtained in the post-April 16 period, but this result is not statistically significant. If we

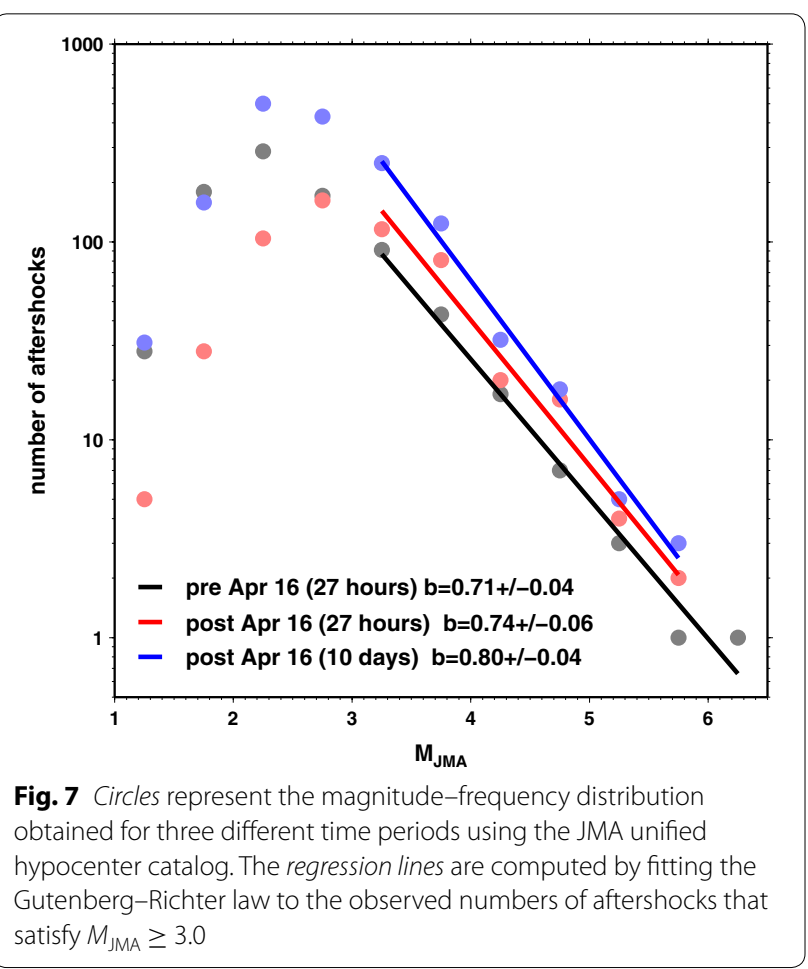


remove the $M_{\mathrm{IMA}} 6.4$ earthquake (only $M>6$ aftershock in all the time periods) that occurred $2.6 \mathrm{~h}$ after the April 14 event, the $b$ value becomes $0.78 \pm 0.02$ in the preApril 16 period. Some studies reported that the $b$ value is significantly smaller for foreshocks than for background seismicity (e.g., Molchan et al. 1999). However, our analysis does not detect such trend significantly.

Figure 8 shows time-lapse decay of number of earthquakes that satisfy $M_{\mathrm{JMA}} \geq 3.0$ in the three time periods. Applying the least-squares method to the number of earthquakes, we obtain $p=1.13 \pm 0.11, p=0.89 \pm 0.12$, and $p=1.07 \pm 0.09$ in the pre-April 16 period (black line), the post-April 16 period for $27 \mathrm{~h}$ long (red line), and the post-April 16 period for 10 days long (blue line), respectively. The $p$ value obtained in the post-April 16 period $(27 \mathrm{~h})$ is smaller than that obtained in other time periods. Because the magnitude of detection threshold may be larger than 3.0 in the lapse times earlier than about 0.1 day, the difference of $p$ value obtained at different time periods could be biased to some extent. However, we note that the slope of energy release rate, which is not affected by the magnitude threshold, is also steeper for the pre-April 16 period than for the post-April 16 period (see Fig. 4). Substituting the estimated $b$ and $p$ values and $\beta=1.4$ (obtained for $3.0 \leq M_{\mathrm{JMA}}<6.5$ ) in the theoretical $p_{\mathrm{E}}=\beta p / b$, we obtain $p_{\mathrm{E}}=2.2, p_{\mathrm{E}}=1.7$, and $p_{\mathrm{E}}=1.9$ in the pre-April 16 period, the post-April 16 period for $27 \mathrm{~h}$ long, and the post-April 16 period for 10 days long, respectively. These results are consistent

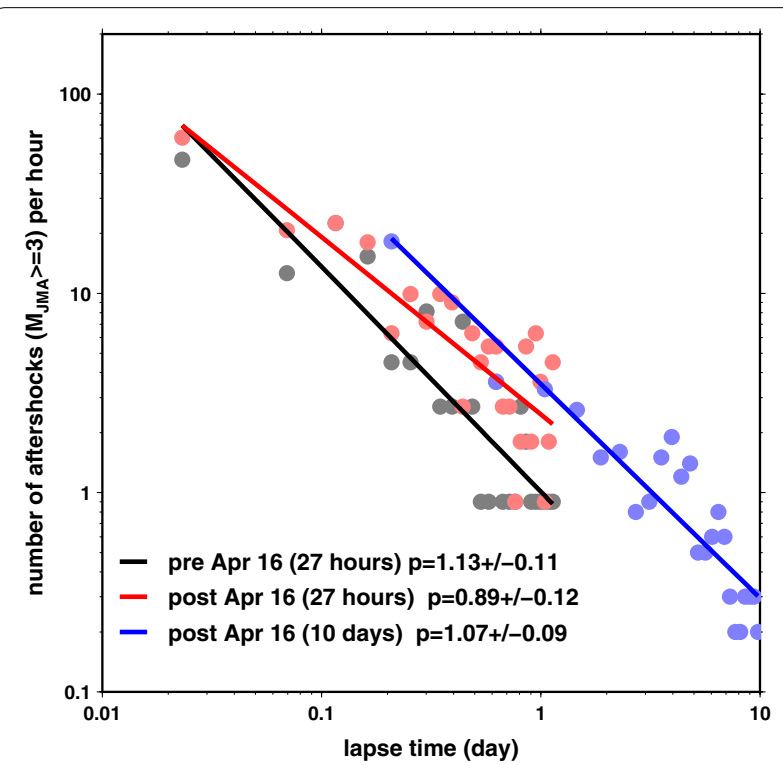

Fig. 8 Circles represent the time-lapse decay of the number of earthquakes that satisfy $M_{\mathrm{JMA}} \geq 3.0$ in three different time periods. The regression lines are computed by fitting the Omori-Utsu law to the observed numbers of aftershocks with the estimated $p_{\mathrm{E}}$ values shown in Fig. 4 for all the time periods within the error bar. This result indicates that the theoretical relationship of $p_{\mathrm{E}}=\beta \mathrm{p} / b$ is supported from the observed seismograms and the earthquake catalog.

\section{Normalized cumulative energy release}

To look further on the difference in the energy releases in the pre- and the post-April 16 periods, we show the cumulative energy releases from the aftershocks occurred in each period in Fig. 9. Figure 9a demonstrates that the amount of cumulative energy release in the pre-April 16 period is slightly smaller than that in the post-April 16 period at the lapse time of $27 \mathrm{~h}$. Figure $9 \mathrm{~b}$ is the similar figure, but the cumulative energy releases in the pre- and the post-April 16 periods are normalized by the energy releases of their "mainshock": $2.7 \times 10^{12} \mathrm{~J}$ and $2.1 \times 10^{13} \mathrm{~J}$ for the April 14 and the April 16 events, respectively. Hereafter, we call this normalized cumulative energy release as NCER. This figure demonstrates that the cumulative energy release in the pre-April 16 period reaches $60 \%$ of that by the April 14 event by the lapse time of $27 \mathrm{~h}$. Although about half of the cumulative energy release is due to the $M_{\mathrm{IMA}} 6.4$ earthquake occurred $2.6 \mathrm{~h}$ after the April 14 event, the NCER still exceeds $30 \%$ even after removal of contribution of this earthquake. On the other hand, the cumulative energy release in the post-April 16 period reaches only 11 and $13 \%$ of that by the April 16 event by the lapse times of $27 \mathrm{~h}$ and 10 days, respectively. The estimated NCER indicates that the April $14 M_{\mathrm{IMA}} 6.5$ event is followed by much larger relative aftershock activity for its magnitude than the April $16 M_{\mathrm{IMA}} 7.3$ event.

Monitoring of NCER would provide information that contributes to judge the ongoing seismicity pattern after the large earthquake. The seismicity pattern would be categorized to mainshock-aftershock type if NCER is below a certain threshold value, while it could be categorized as foreshock-mainshock-aftershock type or swarm type if NCER is above the threshold value. It is interesting to examine the threshold value of NCER that distinguishes the mainshock-aftershock type and other types.

Importantly, the proposed envelope inversion analysis is available in quasi real-time once continuous seismograms are provided, which may be especially useful at regions covered by dense real-time seismograph networks. Through this method, we are able to obtain the $p_{\mathrm{E}}$ value, which is related to $b$ and $p$ values that characterize activity of aftershocks without disturbance by the detection threshold. The NCER value is also important to understand how the energy release process varies depending on seismicity patterns such as mainshockaftershock, foreshock-mainshock-aftershock, and 
a

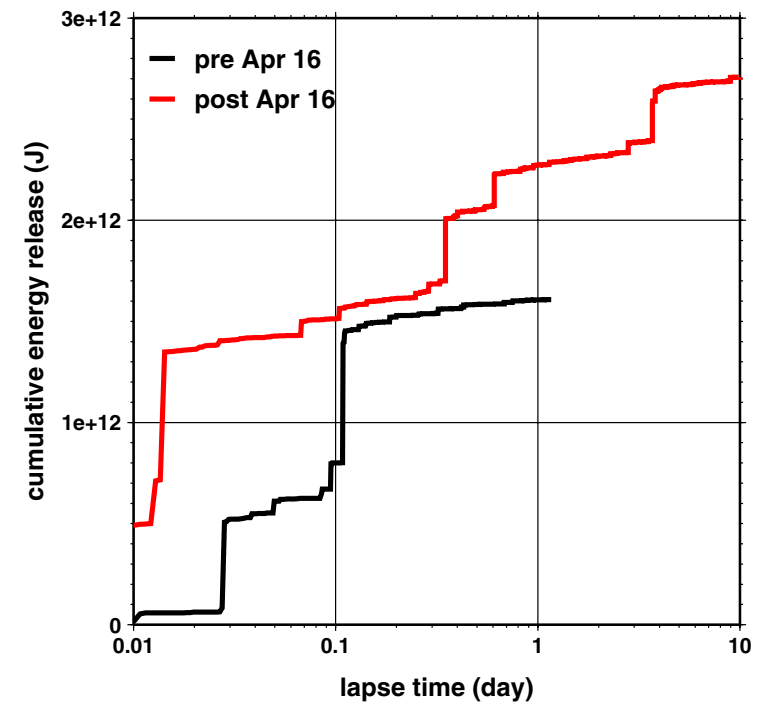

b

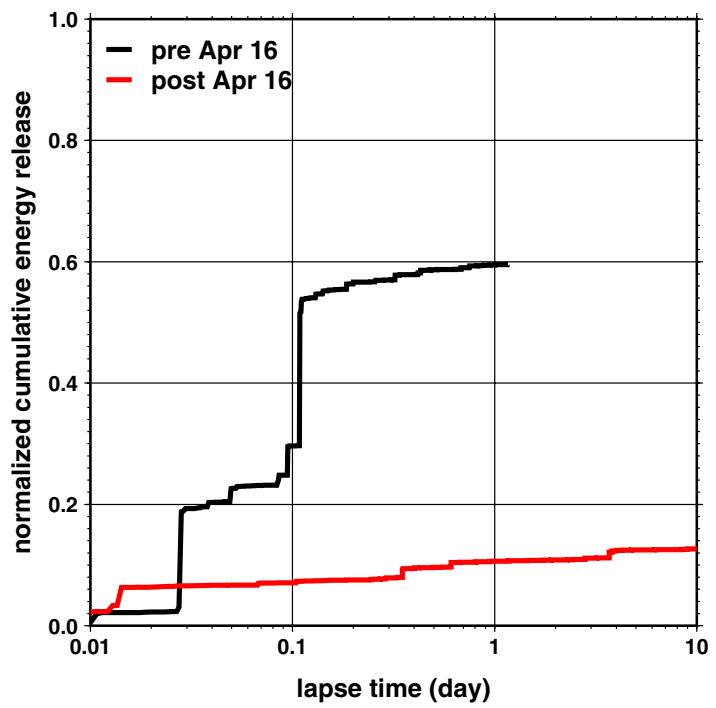

Fig. 9 a Cumulative energy releases $(4-20 \mathrm{~Hz}$ ) by the aftershocks occurring in the pre (black)- and the post (red)-April 16 periods. b Similar plot as a except that they are normalized by the energy releases from the April $14\left(2.7 \times 10^{12} \mathrm{~J}\right)$ and the April $16\left(2.1 \times 10^{13} \mathrm{~J}\right)$ events, respectively

swarm types. It is important to accumulate case studies by applying the envelope inversion analysis to various large earthquakes, especially to those accompanied by large foreshocks.

So far, we do not have any definite reasons that can explain why the aftershock productivity is so different for the April 14 and the April 16 events. Because the main ruptures of these two earthquakes occurred along the different faults (Hinagu and Futagawa faults, respectively) and the faulting processes are also largely different (e.g., Asano and Iwata 2016), the observed different behavior in the aftershock activities is not surprising. It is also important to examine how the aftershock productivity is related to rupture process of the mainshock, geological structure such as fault segmentation, small-scale velocity heterogeneity, intrinsic absorption, and so on.

\section{Conclusion}

In this study, we apply the envelope inversion scheme to the Hi-net continuous records to estimate spatiotemporal high-frequency $(4-20 \mathrm{~Hz})$ energy release associated with the 2016 Kumamoto earthquake sequence. The applied method is useful to estimate a gross feature of the earthquake sequence occurring immediately after a large earthquake, for which conventional earthquake relocation technique based on the phase picking does not work well. We especially focus on the difference in the energy releases after the April $14 M_{\mathrm{JMA}} 6.5$ and the
April $16 M_{\mathrm{JMA}} 7.3$ earthquakes. There is a log-linear relationship between $M_{\mathrm{JMA}}$ and the $4-$ to $20-\mathrm{Hz}$ energy release when $M_{\mathrm{JMA}}$ is smaller than about 4.5, while the relationship does not describe well for larger events probably because $M_{\mathrm{JMA}}$ is determined using lower frequencies. The location of energy release expands to the northeast regions (Aso district and Oita prefecture) after the April 16 event, which includes an M5.6 earthquake triggered in Oita prefecture $34 \mathrm{~s}$ after the April 16 earthquake. The time-lapse decay of the energy release rate obeys a power-law function, where the exponent $p_{E}$ of the power-law decay is estimated to be 1.7-2.1. From combination of the Omori-Utsu law, the GutenbergRichter law, and the magnitude-energy release relationship, we derive the equality given by $p_{E}=\beta p / b$. The $\beta$, $p$, and $b$ values obtained by the analysis of aftershock catalog match the estimated $p_{E}$ values well. The normalized cumulative energy releases (NCERs) in the pre- and post-April 16 periods reach 60 and 11\%, respectively, by the lapse time of $27 \mathrm{~h}$. This discrepancy in NCER indicates that the April $14 M_{\mathrm{JMA}} 6.5$ event was followed by much larger relative energy release for its magnitude than the April $16 M_{\mathrm{JMA}} 7.3$ event. Thus, NCER would reflect the relative productivity of aftershocks and provide information of the ongoing seismicity pattern: mainshock-aftershock type, foreshock-mainshockaftershock type, and swarm type, where the latter may give higher NCER. 


\section{Authors' contributions}

KS analyzed the data and drafted the manuscript. HN and KS provided many comments and suggestions to improve the manuscript. All authors read and approved the final manuscript.

\section{Author details \\ ${ }^{1}$ National Research Institute for Earth Science and Disaster Resilience, Tsukuba, Japan. ${ }^{2}$ Tohoku University, Sendai, Japan.}

\section{Acknowledgements}

We thank Japan Meteorological Agency (JMA) and corresponding universities and institutes that contribute to compiling the JMA unified hypocenter catalog. We also thank Dr. M. Hashimoto, editor of Earth Planets and Space, two anonymous reviewers, and one guest editor for their thoughtful comments to improve our manuscript. Seismic Analysis Code (SAC) and Generic Mapping Tools (GMT) were used for signal processing and figure plotting, respectively. This work was partly supported by the Earthquake Research Institute Cooperative Research Program (2015-B-01).

\section{Competing interests}

The authors declare that they have no competing interests.

\section{Data}

The Hi-net and KiK-net records are available through the webpage of NIED (http://www.hinet.bosai.go.jp/?LANG=en and http://www.kyoshin.bosai. go.jp/, respectively). The JMA unified hypocenter catalog is available from http://www.data.jma.go.jp/svd/egev/data/bulletin/hypo.html.

\section{Appendix 1: Synthesis of the hybrid theoretical envelope}

In Sawazaki and Enescu (2014), the envelope Green's function is computed on the basis of the approximate solution of the radiative transfer equation for a 3-D isotopic scattering media (Paasschens 1997) given by

$$
\begin{aligned}
& G_{C}(r, t) \approx \frac{1}{4 \pi V r^{2}} \delta\left(t-\frac{r}{V}\right) \exp \left\{-\left[g_{0} V+Q_{i}^{-1} \omega\right] t\right\} \\
&+ \frac{\left[1-r^{2} /(V t)^{2}\right]^{1 / 8}}{\left[4 \pi V t /\left(3 g_{0}\right)\right]^{3 / 2}} \exp \left\{-\left[g_{0} V+Q_{i}^{-1} \omega\right] t\right\} \\
& \times M\left[g_{0} V t\left(1-\frac{r^{2}}{V^{2} t^{2}}\right)^{3 / 4}\right] H\left(t-\frac{r}{V}\right) \\
& M(x) \approx \exp (x) \sqrt{1+2.026 / x}
\end{aligned}
$$

where $r, V, g_{0}$, and $Q_{i}^{-1}$ represent the source-receiver distance, the background $\mathrm{S}$-wave velocity, the (isotropic) scattering coefficient, and the inverse of intrinsic absorption Q factor, respectively. Equation (10) is suitable to describe the coda-wave envelope, while it cannot describe the direct-wave envelope well because the direct wave is composed of the wavefield experienced multiple forward scattering. Because we use direct-wave part of the envelope to estimate location of the energy release, using Eq. (10) as envelope Green's function may cause a large error in estimation of the location.
On the other hand, solution of the parabolic wave equation for a 3-D Gaussian-type random inhomogeneous media (Shishov 1974) is given by

$$
\begin{aligned}
G_{D}(r, t)= & \frac{\pi}{8 r^{2} t_{M} V} H\left(t-\frac{r}{V}\right) \\
& \sum_{n=1}^{\infty}(-1)^{n+1} n^{2} \exp \left[-n^{2} \pi^{2} \frac{t-r / V}{4 t_{M}}-Q_{i}^{-1} \omega t\right], \\
& t_{M}=\frac{\sqrt{\pi} \varepsilon^{2} r^{2}}{2 a V}
\end{aligned}
$$

where $a$ and $\varepsilon$ represent the correlation length and the root-mean-squared fractional velocity fluctuation of the random media, respectively. The original formulation by Shishov (1974) is divided by the background velocity $V$ in Eq. (11) in order to replace the original physical dimension of unit energy flux $\left(1 / \mathrm{m}^{2} \mathrm{~s}\right)$ with the dimension of unit energy density $\left(1 / \mathrm{m}^{3}\right)$. Equation (11) is suitable to describe the direct-wave envelope because the parabolic wave equation controls the wave propagation which experiences multiple forward scattering. Although the Gaussian-type random media adopted in Eq. (11) is not suitable to represent the power-law spectrum recognized in subsurface of the Earth (e.g., Sato et al. 2012), we use Eq. (11) to gain an advantage from the analytical solution.

By using Eqs. (11) and (10) to describe the direct- and the coda-wave envelopes, respectively, we synthesize the hybrid envelope which can describe the whole envelope shape. Following Saito et al. (2003), we synthesize the hybrid envelope using the formula given by

$$
G_{H}(r, t)=G_{D}(r, t) e^{-g_{L} V t}+G_{C}^{\prime}(r, t),
$$

where $G_{C}^{\prime}$ is equal to $G_{C}$ of Eq. (10) except that the ballistic term (the first term of r.h.s. of Eq. 10) is subtracted. The parameter $g_{L}=7 \times 10^{-3} \mathrm{~km}^{-1}$ is selected so that the spatial integral of the total energy is conserved for an undamped medium. In addition to the $\mathrm{S}$-wave envelope, we also compute the P-wave envelope by using the same parameters except that $V$ is regarded as the P-wave velocity. To correct for the difference in the $\mathrm{P}$ - and the $\mathrm{S}$-wave energy releases by a point sheardislocation source, we multiply $2 V_{\mathrm{S}}^{5} / 3 V_{\mathrm{P}}^{5}=0.041$ to the synthesized P-wave envelope (Aki and Richards 2002). Figure 10 shows an example of the synthesized hybrid envelope $G_{H}$ together with $G_{D}$ and $G_{C}$, where $G_{H}$ can better describe the observed envelope than either of $G_{D}$ and $G_{C}$. 


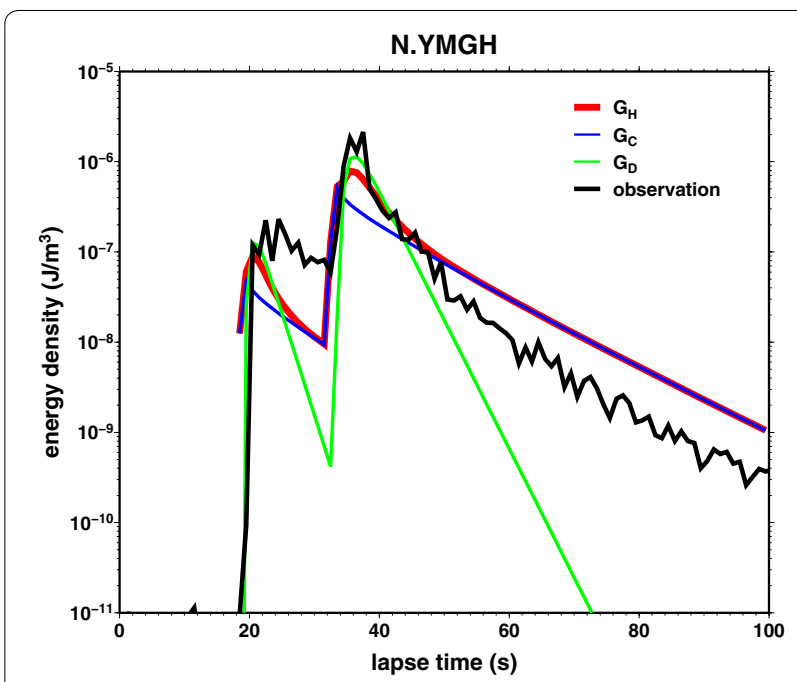

Fig. 10 Examples of the hybrid theoretical envelope (red), the synthesized envelopes on the basis of the isotropic scattering model (blue), and the forward scattering approximation (green). The black curve shows the observed seismogram envelope at station N.YMGH for an earthquake occurring at $115 \mathrm{~km}$ distance

\section{Appendix 2: Scheme to estimate energy release location}

The location of energy release was not constrained well in the method by Sawazaki and Enescu (2014) because they used peak amplitude (amplitude of ballistic S-wave) of the envelope to determine the energy release location. Because the observed peak amplitude is affected significantly not only by the source-receiver distance but also by the radiation pattern, the local intrinsic absorption, and so on, which are not reflected in the theoretical envelope, the energy release location estimated from the theoretical peak amplitudes tends to include large error, especially when the station coverage is poor.

Because the peak arrival time of the envelope is less affected by the radiation pattern and the local intrinsic absorption than the peak amplitude (e.g., Sawazaki et al. 2011), we use the theoretical peak arrival time at each station to locate the energy release point in this study. First, we compute the theoretical peak arrival times for each source-receiver pair using the hybrid theoretical envelope given by Eq. (12). Then we compute the station-average of the observed log-amplitudes recorded at the theoretical peak arrival times. If the tentatively selected source-grid is closer to the actual energy release location, the observed amplitude recorded at the theoretical peak arrival time becomes larger, and the station-average of the log-amplitude also becomes larger. Therefore, we can regard the source-grid that gives the largest station-averaged log-amplitude as the location of the energy release.
Received: 25 July 2016 Accepted: 29 October 2016

Published online: 23 November 2016

\section{References}

Aki K, Richards PG (2002) Quantitative seismology. Univ Sci Books, Sausalito

Asano K, Iwata T (2016) Source rupture processes of the foreshock and mainshock in the 2016 Kumamoto earthquake sequence estimated from the kinematic waveform inversion of strong motion data. Earth Planets Space 68:147

Cabinet Office, Government of Japan (2016) http://www.bousai.go.jp/ updates/h280April14jishin/. Accessed 14 June 2016 (in Japanese)

Carcolé E, Sato H (2010) Spatial distribution of scattering loss and intrinsic absorption of short-period $S$ waves in the lithosphere of Japan on the basis of the Multiple Lapse Time Window Analysis of Hi-net data. Geophys J Int 180(1):268-290

Enescu B, Mori J, Miyazawa M, Kano Y (2009) Omori-Utsu law c-values associated with recent moderate earthquakes in Japan. Bull Seism Soc Am 99(2A):884-891

Eshelby JD (1957) The determination of the elastic field of an ellipsoidal inclusion, and related problems. Proc R Soc Lond A Math Phys Eng Sci 241(1226):376-396

Gutenberg B, Richter CF (1944) Frequency of earthquakes in California. Bull Seism Soc Am 34(4):185-188

Lippiello E, Cirillo A, Godano G, Papadimitriou E, Karakostas V (2016) Real-time forecast of aftershocks from a single seismic station signal. Geophys Res Lett 43(12):6252-6258

Madariaga R (1976) Dynamics of an expanding circular fault. Bull Seism Soc Am 66(3):639-666

Molchan GM, Kronrod TL, Nekrasova AK (1999) Immediate foreshocks: time variation of the b-value. Phys Earth Planet Inter 111(3):229-240

Obara K, Kasahara K, Hori S, Okada Y (2005) A densely distributed highsensitivity seismograph network in Japan: Hi-net by National Research Institute for Earth Science and Disaster Prevention. Rev Scient Instrum 76(2):021301

Okada Y, Kasahara K, Hori S, Obara K, Sekiguchi S, Fujiwara H, Yamamoto A (2004) Recent progress of seismic observation networks in Japan -Hi-net, F-net, K-net and KiK-net-. Earth Planets Space 56:15-28

Omi T, Ogata Y, Hirata Y, Aihara K (2015) Intermediate-term forecasting of aftershocks from an early aftershock sequence: Bayesian and ensemble forecasting approaches. J Geophys Res 120(4):2561-2578

Omi T, Ogata Y, Shiomi K, Enescu B, Sawazaki K, Aihara K (2016) Automatic aftershock forecasting: a forecast test of aftershocks using the real-time data in Japan. Bull Seism Soc Am. doi:1 0.1785/0120160100

Paasschens JCJ (1997) Solution of the time-dependent Boltzmann equation. Phys Rev E 56(1):1135-1141

Phillips W, Aki K (1986) Site amplification of coda waves from local earthquakes in central California. Bull Seism Soc Am 76(3):627-648

Saito T, Sato H, Fehler M, Ohtake M (2003) Simulating the envelope of scalar waves in 2D random media having power-law spectra of velocity fluctuation. Bull Seism Soc Am 93(1):240-252

Sato H, Fehler MC, Maeda T (2012) Seismic wave propagation and scattering in the heterogeneous earth. Springer, Berlin

Sawazaki K (2016) Rapid detection of early aftershocks using high-frequency seismogram envelope: improvement of location estimation of energy radiation point. Japan Geophysical Union: SSS29-06 (in Japanese)

Sawazaki K, Enescu B (2014) Imaging the high-frequency energy radiation process of a main shock and its early aftershock sequence: the case of the 2008 Iwate-Miyagi Nairiku earthquake, Japan. J Geophys Res 119(6):4729-4746

Sawazaki K, Sato H, Nishimura T (2011) Envelope synthesis of short-period seismograms in 3-D random media for a point shear dislocation source based on the forward scattering approximation: application to small strike-slip earthquakes in southwestern Japan. J Geophys Res 116(B8)

Shiomi K, Obara K, Kasahara K (2005) Amplitude saturation of the NIED Hi-net waveforms and simple criteria for recognition. Zisin 2 57:451-461 (in Japanese)

Shishov VI (1974) Effect of refraction on scintillation characteristics and average pulse shape of pulsars. Soviet Astron 17:598

Utsu T (1961) A statistical study on the occurrence of aftershocks. Geophys Mag 30:521-605

Utsu T (2001) Seismology, Kyoritsu (in Japanese), Tokyo 